\title{
Late Fusion of Bayesian and Convolutional Models for Action Recognition
}

\author{
Camille Maurice \\ LAAS-CNRS \\ Toulouse, France \\ Email: cmaurice@laas.fr
}

\author{
Francisco Madrigal \\ LAAS-CNRS \\ Toulouse, France \\ Email: jfmadrigal@laas.fr
}

\author{
Frédéric Lerasle \\ LAAS-CNRS, University Paul Sabatier \\ Toulouse, France \\ Email: lerasle@laas.fr
}

\begin{abstract}
The activities we do in our daily-life are generally carried out as a succession of atomic actions, following a logical order. During a video sequence, actions usually follow a logical order. In this paper, we propose a hybrid approach resulting from the fusion of a deep learning neural network with a Bayesianbased approach. The latter models human-object interactions and transition between actions. The key idea is to combine both approaches in the final prediction. We validate our strategy in two public datasets: CAD-120 and Watch-n-Patch. We show that our fusion approach yields performance gains in accuracy of respectively +4 percentage points $(\mathrm{pp})$ and +6 pp over a baseline approach. Temporal action recognition performances are clearly improved by the fusion, especially when classes are imbalanced.
\end{abstract}

\section{INTRODUCTION}

The recognition of human activities is at the core of the development of many practical applications such as monitoring of domestic activities or human-robot collaboration. An activity is defined by successive time sequences of actions [1], [2] e.g.: prepare coffee involves the successive actions pour in water, add ground coffee and start the machine. On the one hand, activities performed by humans in a domestic or industrial environment can be very different, for example in the nature of the objects involved. On the other hand, the atomic actions performed may be similar in any context. Indeed, these atomic actions concern the movement of objects, their capture, or the interactions they may have with their environment. Therefore, we are interested in the recognition of atomic actions and their sequencing because higher level activities can be represented by atomic actions arranged in sequences following a logical order.

Data-driven approaches based on convolutional neural networks $(\mathrm{CNN})$ adapted to the video domain with 3D convolutions allow the recognition of actions in video streams. 3D convolutional neural networks learn spatio-temporal features simultaneously. Approaches like C3D [3] obtain an accuracy of $90.4 \%$ in the action recognition dataset UCF101 [4]. However, 3D convolutions increase the size of the network and thus the number of parameters to be learned (i.e., $17 \mathrm{M}$ with $\mathrm{C} 3 \mathrm{D}$ ). As any $\mathrm{CNN}$, they require a lot of annotated data, hence the emergence of larger annotated datasets such as NTU RGB+D [5], UCF101 [4] and Kinetic [6]. For example UCF101 contains 27 hours of videos and NTU RGB+D [5] 56880 clips. Despite these advances, action recognition is still
Fig. 1: Different individual approaches (1) (2) (3) and their fusion (4). During training, the trained layers are represented in green.

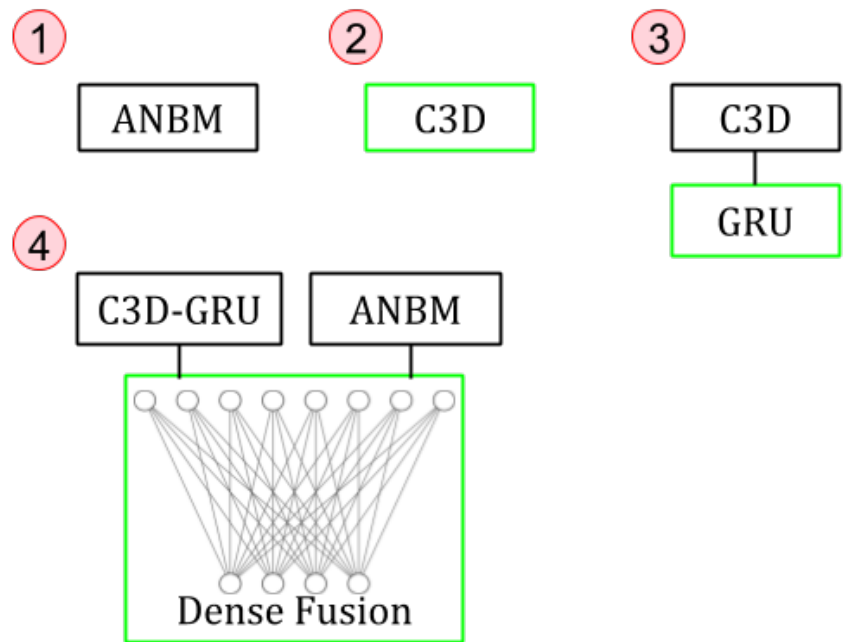

a challenge because 3D convolution networks only aggregate temporal features on video clips i.e. pre-segmented actions without temporal relations between those clips. They are taken independently and do not take into account the temporal logic in a sequence of actions.

Often, large datasets like Kinetic [6] and UCF101 [4] are created from videos collected on YouTube. The different classes are performed in radically different environments, for example swimming vs. playing guitar. However in the context of monitoring domestic activities, the actions to detect take place in a similar environment and have a temporal coherence in their sequence representing a certain activity. Recognition of sequential actions with low inter-class variance, imbalanced classes, and/or under-represented classes is still a challenge for conventional convolution networks.

Historically, probabilistic-based approaches [7], [8] propose to characterize the actions in a more explicit way through modeling the observations of the scene elements: human pose, objects and their interaction through time. These approaches usually based on probabilistic models generally offer lower 
performance compared to convolutional networks. Nevertheless, they generally require less data because they also have fewer underlying free parameters to tune. Therefore their interpretability is less dependent on the available learning data (e.g. less subject to over-fitting). These approaches are relevant in the case of a small number of samples available for training. For example, our previous Bayesian approach for action recognition ANBM (for A New Bayesian Model [9]), models both the interactions between objects and humanobjects through about 50 parameters. Let us note that our ANBM approach also takes into account the transitions between different actions in order to ensure temporal consistency throughout the sequence of actions.

Building on the observation of a possible synergy of the two approaches, we propose a hybrid framework with a fusion at the decision level, of a C3D [3] convolutional network and our probabilistic ANBM [9] approach based on explicit humanobject observations. These two approaches take into account the spatio-temporal characteristics of the different classes of actions. Due to the large number of parameters, the C3D network needs a lot of annotated data to be relevant since learning is difficult in the case of under-represented classes. The ANBM approach depends on handcrafted models and even with a little data the prediction of under-represented classes is possible.

Thus, our contributions are: (1) one first minor contribution is the addition of a Gated Recurrent Unit (GRU) recurrent layer to the $\mathrm{C} 3 \mathrm{D}$ architecture for action recognition which also models the temporal correlations between actions, (2) the comparison of both approaches (ANBM and C3D-GRU) on two public datasets CAD-120 and Watch-n-Patch, (3) implementation and evaluation of a late fusion mechanism of the predictions of these two approaches and comparison with the literature. We observe a performance gain from this hybrid approach.

The article is organized as follows. In section 2 we present the state of the art and the context of our work. Then in section 3 we present our hybrid approach for action detection. A comparative study of our results is presented in section 4 . Finally, section 5 presents our conclusion and future prospects.

\section{STATE OF THE ART}

The recognition of static actions on single image can be done by localizing certain objects in an image, i.e., Zhou et al. [10] or Oquab et al. [11]. This kind of approach has been popularized by the Pascal VOC 2012 challenge, where the goal is to recognize actions in images [12]. While this is relevant when the classes of actions to be recognized occur in different environments, these approaches are inappropriate for recognizing successive atomic actions occurring in a sequence of action taking place in the same scene. It is movements and objects involved in the execution of an action that allow it to be discriminated, for example when opening or closing a door. This is why we focus on approaches using spatio-temporal information from videos in order to consider the dynamics of gestures and objects during action classification.
Historical approaches perform dynamic action recognition through probabilistic modeling of the observations involved. In addition, these model-based approaches may include trajectory models for human pose, information of the spatial configuration of the objects in the scene or their affordance [13]. Li et al. [7] propose the use of Gaussian mixture to recognize different actions in the MSRAction dataset [7]. Koppula and Saxena [8] propose the use of conditional random fields (CRFs) to model the scene and the spatio-temporal relationships that appear in CAD-120 [8]. More recently, we have proposed a new Bayesian ANBM [9] approach based on explicit 3D modeling of contextual features, both spatially and temporally. These approaches rely on a smaller number of parameters than those of C3D networks. In fact, they require less data and are evaluated on datasets that are generally smaller. For example MSRAction [7] contains 420 sequences and CAD-120 [8] contains 120 videos for about 1000 clips after segmentation of the actions. They also have the advantage of being more interpretable than CNN approaches.

One of the challenges with convolutional networks is their dependency to the amount of data available for training. Learning their many parameters is based on the amount of data available for training. The introduction of 3D [14] convolution filters allows to simultaneously extract spatiotemporal descriptors from a set of frames representing an action, called a clip. These descriptors are appropriate for implicitly capturing the context related to the video content. This idea has been taken up by C3D [3] and other variants [15], [16], [17] for action detection. Adding video clips at the input of the network requires increasing its size compared to its 2D CNN counterpart. C3D networks extract a global descriptor from the clip independently of the action that took place previously. This is particularly suitable and shows strong results for large-scale datasets with many small clips such as UCF101 [4] with its 13000 clips and an average duration of 7 seconds. These arrays only aggregate temporal information over a fixed window size, typically 16 frames. This is not suitable for recognizing actions that have temporal consistency within their sequencing.

Hence the interest in adding a recurrent layer to a 3Dconvolutional network. Wang et al. [18] propose to add a Long Short Term Memory layer (LSTM) to such a network. Also in [19], [20] the authors propose to either add a LSTM-layer or a GRU-layer to reinforce the temporal coherence within the action clip and evaluate themselves on UCF101 for example. Instead, we propose to add logical consistency in the actions sequencing.

The fusion of C3D networks with other modalities has already improved its performance in various challenges of the Computer Vision community. For example, space-time fusion [21] consists in merging an image with an optical flow sequence that describes motion. This improves the performance in comparison to a C3D network alone, which seeks to simultaneously extract temporal and spatial features at the 3D convolution layers. There are also methods that propose a fusion of different features of different nature such as 
audio and video [22]. These different approaches show the advantages of using a fusion mechanism to increase overall performance. However, this gain is achieved at the expense of the amount of data required for training. The addition of more modalities increases the number of parameters to be learned for the convolution network. This has two effects: first it required the existence of a such dataset, and second it increases the training time. A late fusion is proposed by [23] for pose attention in RGB videos.

We propose to merge two spatio-temporal approaches, one based on context modeling via learning such as C3D [3] and our ANBM [9] approach based on Bayesian models and 3D human and objects observations of the scene. This fusion is not done at the feature level but later at their predictions level towards the same layer. We propose to merge them using a fully connected layer, i.e. dense layer. Only a few works study the late fusion of two classes of approaches that a priori complement each other and the gains that this can yield.

Public datasets such as Watch-n-Patch [24] and CAD120 [8] allow to evaluate the recognition of atomic actions. These datasets offer approximately 20 -seconds long videos in which different atomic actions are annotated. The actions follow each other in a logical order, for example we cannot move an object that has not been previously captured. In these datasets, the sequences of actions are more or less correlated. Moreover some classes are under-represented in these datasets, which is generally a lock for C3D learning.

\section{PRoposed ApProACH}

In this section we describe the proposed architecture for the fusion of probabilities predicted by the ANBM [9] Bayesian approach with those of the modified C3D [3] network. We recall our previous ANBM approach in Section III-A and then briefly describe the C3D network in section IIII-B and its modification (C3D-GRU) in Section III-C. Section III-D details the proposed late fusion strategy.

\section{A. Bayesian Approach With Human-Object Observations}

This approach [9] is based on the following insights: human pose, human-object and object-to-object interactions, performed during the execution of an action, provide spatiotemporal information that allows the recognition of the ongoing action. Moreover, it considers temporal information such as transition between actions during a sequence. We have modeled these observations in order to be able to estimate, at each time of the video, the probabilities of each considered actions.

All the elements of the scene are first localized in the image plane by 2D state-of-the-art detectors one for human pose estimation an another for the objects. Then they are modeled in 3D space using RGB-D sensor (e.g. Kinect) calibration data. The detection of the human pose in the image is based on OpenPose [25], which is trained on MSCOCO Keypoints Challenge [26]. We use Single Shot Multi-Box Detector (SSD) [27] to recognize objects, which is trained with the MSCOCO dataset [26].
Each action $a$ is associated to a model. Let $\mathrm{A}=$ $\left\{a^{1}, a^{2}, \ldots, a^{N}\right\}$ be the set of $N$ actions. The joint observation of the human pose $s_{t}$ and the set of objects $\Omega_{t}$ is described at time $t$ by $O_{t}=\left\{s_{t}, \Omega_{t}\right\}$ where $\Omega_{t}=\left\{\omega^{1}, \omega^{2}, \ldots, \omega^{\operatorname{Card}(\Omega)}\right\}$ with $\operatorname{Card}(\Omega)$ being the number of objects in the scene. The inference is performed on a sliding window of $T$ frames, so that this approach does not require video clips segmentation beforehand, and ensure temporal consistency of the observations. We model the a posteriori probability of the actions given the observations as follows:

$$
p\left(a_{0: T} \mid O_{0: T}\right) \propto \prod_{t=0}^{T} p\left(O_{t} \mid a_{t}\right) \prod_{t=1}^{T} p\left(a_{t} \mid a_{t-1}\right) .
$$

Where $p\left(O_{t} \mid a_{t}\right)$ is the likelihood of the observation given the action $a_{t}$. The term $p\left(a_{t} \mid a_{t-1}\right)$ characterizes the probabilities of transitions between two successive actions. All the observations of the scene in this approach are modelled in 3D. Objects and pose $2 \mathrm{D}$ coordinates are projected onto the $3 \mathrm{D}$ space thanks to the sensor calibration data. It allows ANBM to be more robust to changes of point of view than an approach based solely on 2D spatial characteristics. We invite the reader to consult [9] the paper for more in-depth details.

\section{B. $3 D$ convolution network: $C 3 D$}

C3D [3] is a deep learning network that takes into account, in addition to images, a third dimension corresponding to time. The architecture includes $3 \times 3 \times 3$ convolution filters, followed by $2 \times 2 \times 2$ pooling layers. The introduction of $3 \mathrm{D}$ convolution filters allows to learn spatio-temporal descriptors from a video stream.

On the one hand, they provide a compact (4096) description of a video stream of size $\mathrm{H} \mathrm{x} \mathrm{W} \mathrm{x} \mathrm{C} \mathrm{x} \mathrm{L.} \mathrm{With} \mathrm{L}$ the length of the video clip containing the action, usually 16 frames. These networks are able to learn those spatio-temporal descriptors implicitly. Like other networks they perform end-to-end learning without expert information (unlike any probabilistic approach e.g. ANBM).

On the other hand, given the millions of parameters to be learned, poorly represented classes are hardly well recognized and it is more difficult to predict them correctly. The action must be sampled on 16 frames in the original implementation, of course it is possible to enlarge this time window but it requires more memory. Tran et al [3] also offer a sliding window system of descriptor averaging. However, in all cases, C3D requires a pre-segmentation of actions in sequences, which does not make it a suitable method for online action recognition. Moreover, there are no mechanisms to take into account the temporal context in which the video clip is inserted during training. Therefore there is no consideration of the previous action.

\section{Adding a recurrent layer: $C 3 D-G R U$}

In order to compensate the lack of a mechanism that ensure the temporal consistency along the sequence, across the videoclips. We propose to take into account the previous action in the detection of the current action by adding a recurrent layer. 
Fig. 2: An action sequence from CAD-120 [8] dataset: actor 1, video 2305260828, action microwaving-food. From left to right : reach, open, reach, move, place. In blue: human pose detected by OpenPose. In yellow: objects detected by SSD.

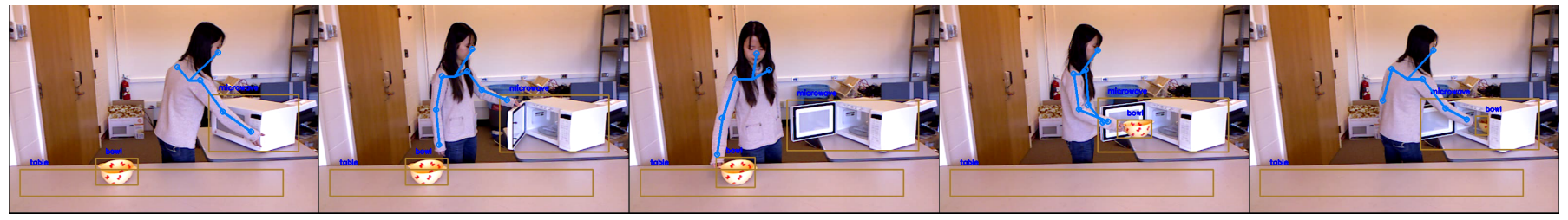

Once we trained C3D, we retrieve its weights, freeze them and add a recurrent GRU-type layer. C3D is trained with data augmentation that is not able to perform in the same manner for the GRU-type layer. Indeed we need to preserve temporal coherence and segments to train in logic order.

Then we adapt the GRU layer to take into account two successive clips corresponding to two different, but successive, actions. We do not re-train the whole C3D network but we only perform a fine-tuning at the level of the last layers. To illustrate the importance of the nature of the previous actions we notice that among all the possible transitions between any two pairs of actions in Watch-n-Patch [8], only about $20 \%$ are actually occurring. By adding this extra constraint while training the GRU-layer, we hope to reduce the number of false positive detections of some classes. This strategy is illustrated in Fig. 1 . number 3 . We call this approach C3D-GRU afterwards.

\section{Late fusion with a dense layer}

We therefore have two approaches to predict actions from video clips based on spatio-temporal data, explicitly with ANBM and implicitly with C3D-GRU. Both approaches also consider the existing transitions between two successive actions. On the one hand with ANBM we have modeled each action, on the other hand C3D-GRU learns from the datasets, whose classes are not equally distributed. Indeed in the detection of atomic actions, some actions are found more frequently. For example the displacement of an object (moving) represents $34 \%$ of the actions of CAD-120, it mandatory occurs before many different actions such as to drink because we need to move the bottle before. We propose a fusion of their respective predictions. Both approaches estimate probabilities for each class. We have one for ANBM and for C3D-GRU we have a vector corresponding to the output of the soft-max layer.

We propose a strategy that takes as input video clips that are processed through the ANBM approach and also through the C3D-GRU network described above, whose C3D layers weights are frozen. We thus obtain two prediction vectors for each of the approaches that are later concatenated. This concatenation is connected to a dense layer of the same size as the number of classes, as shown with only $N=4$ classes as example in Fig 1. number 4. So there are only $N^{2}+N$ parameters to learn $\left(N^{2}\right.$ weights related to the dense layer and $N$ bias related to activation). This interconnection enables to take the advantage of both approaches in the final decision. We call this approach C3D-GRU-DF thereafter.

\section{EXPERIMENTS AND RESULTS}

\section{A. Public Datasets}

Let us recall that we propose an initial approach to detect actions in [9]. This online approach is able to detect actions sequences of from a video stream and to manage transitions between actions. We wish to take advantage of this asset, so we evaluate ourselves on two public datasets which contain such action sequences: CAD-120 [8] and Watch-n-Patch [24].

a) CAD-120: The CAD-120 [8] dataset consists of 120 videos with RGB-D channels, played by 4 actors. It contains 10 daily life activities (preparing a bowl of cereal, taking medication...). These activities involve 10 actions: reaching, moving, pouring, eating, drinking, placing, opening, closing, null. Here, each video represents an activity as defined in the section I. The inequitable distribution of actions, expressed by the corresponding percentage of frames, is described in Tab. III. An illustration of this dataset is presented in Fig. 2 .

b) Watch-n-Patch: The office environment consists of 196 videos recorded in 8 different offices. There are 10 annotated actions: read, walk, leave-office, fetch-book, putback-book, put-down-item, pick-up-item, play-computer, turnon computer, turn-off computer. Here again some actions are dependent on the action that takes place previously, e.g. to play the computer, the screen must be turned on. Action classes are not equally distributed as shown in Tab. III

TABLE III: Detail of class distribution within datasets and the number of clips.

\begin{tabular}{lll} 
Dataset & Number of clips & Distribution (\% per class) \\
\hline CAD-120 & 1149 & {$[23,30,3,3,3,15,4,3,1,14]$} \\
Watch-n-Patch & 1148 & {$[12,16,21,6,4,14,9,9,5,3]$} \\
\hline
\end{tabular}

\section{B. System Evaluation}

a) Managing ANBM's Predictions: We record the prediction probabilities of ANBM at each frame, then we take their averages over the duration of each action to assign a class to each video clip representing an action.

b) Pre-processing for $C 3 D$ : We keep the original settings of the publication [3] for the input image size by setting it to $112 \times 112$ pixels. The video clips are cropped around the enlarged bounding box containing the actor and objects in the action context. This bounding box is detected using the human pose inferred by OpenPose [25]. This allows the network to 
TABLE I: Results of our different variants on Watch-n-Patch. Performance metrics considered are macro-accuracy (M) and micro-accuracy $(\mu)$.

\begin{tabular}{l|cccccccc|ccccc} 
Architecture & \multicolumn{3}{|c}{ Sample 0 } & \multicolumn{2}{c}{ Sample 1 } & \multicolumn{2}{c}{ Sample 2 } & \multicolumn{2}{c}{ Sample 3 } & \multicolumn{3}{c}{ Mean } & \multicolumn{3}{c}{ Standard Deviation } \\
& $\mu$ & $\mathrm{M}$ & $\mu$ & $\mathrm{M}$ & $\mu$ & $\mathrm{M}$ & $\mu$ & $\mathrm{M}$ & $\mu$ & $\mathrm{M}$ & $\mu$ & $\mathrm{M}$ \\
\hline 1 - ANBM & 0.78 & 0.79 & 0.73 & 0.74 & 0.76 & 0.77 & 0.75 & 0.75 & 0.76 & 0.76 & 0.02 & 0.02 \\
2 - C3D & 0.72 & 0.65 & 0.73 & 0.64 & 0.75 & 0.69 & 0.74 & 0.64 & 0.74 & 0.66 & 0.01 & 0.02 \\
3 - C3D-GRU & 0.89 & 0.87 & 0.86 & 0.77 & 0.85 & 0.77 & 0.89 & 0.84 & 0.87 & 0.81 & 0.02 & 0.05 \\
\hline 4 - C3D-GRU-ANBM-DF & $\mathbf{0 . 9 4}$ & $\mathbf{0 . 9 1}$ & $\mathbf{0 . 9 3}$ & $\mathbf{0 . 9 0}$ & $\mathbf{0 . 9 3}$ & $\mathbf{0 . 9 1}$ & $\mathbf{0 . 9 3}$ & $\mathbf{0 . 8 9}$ & $\mathbf{0 . 9 3}$ & $\mathbf{0 . 9 0}$ & $\mathbf{0 . 0 0 1}$ & $\mathbf{0 . 0 1}$ \\
\hline
\end{tabular}

TABLE II: Results of our different variants on CAD-120. Performance metrics considered are macro-accuracy (M) and microaccuracy $(\mu)$.

\begin{tabular}{l|cccccccc|ccccc} 
Architecture & \multicolumn{2}{|c}{ Actor 1 } & \multicolumn{2}{c}{ Actor 2 } & \multicolumn{2}{c}{ Actor 3 } & \multicolumn{2}{c}{ Actor 4 } & \multicolumn{3}{c}{ Mean } & \multicolumn{2}{c}{ Standard Deviation } \\
& $\mu$ & $\mathrm{M}$ & $\mu$ & $\mathrm{M}$ & $\mu$ & $\mathrm{M}$ & $\mu$ & $\mathrm{M}$ & $\mu$ & $\mathrm{M}$ & $\mu$ & $\mathrm{M}$ \\
\hline 1 - ANBM & 0.84 & 0.77 & 0.78 & 0.81 & 0.82 & 0.76 & 0.82 & 0.77 & 0.82 & 0.78 & 0.03 & 0.02 \\
2 - C3D & 0.58 & 0.45 & 0.70 & 0.61 & 0.64 & 0.57 & 0.56 & 0.35 & 0.62 & 0.50 & 0.06 & 0.12 \\
3 - C3D-GRU & 0.61 & 0.49 & 0.76 & 0.73 & 0.66 & 0.60 & 0.60 & 0.45 & 0.66 & 0.57 & 0.07 & 0.13 \\
\hline 4 - C3D-GRU-ANBM-DF & $\mathbf{0 . 8 6}$ & $\mathbf{0 . 8 0}$ & $\mathbf{0 . 8 9}$ & $\mathbf{0 . 9 1}$ & $\mathbf{0 . 8 4}$ & $\mathbf{0 . 8 2}$ & $\mathbf{0 . 8 3}$ & $\mathbf{0 . 7 9}$ & $\mathbf{0 . 8 6}$ & $\mathbf{0 . 8 3}$ & $\mathbf{0 . 0 3}$ & 0.05 \\
\hline
\end{tabular}

focus its attention on the area where the activity is taking place. The C3D network takes an action sequence of fixed size: 16 frames. In practice, since we consider atomic actions, which are relatively short, we do not use a sliding window on the sequences but rather simply re-sample the sequences.

c) Training: The network weights are trained using a stochastic gradient descent on mini-batches of size 16 with a momentum of 0.9 . We initialize the learning rate to 0.01 and it decreases over time. The training is done on a GeForce GTX 1080 Ti graphics card. We use the cross-entropy categorical loss function.

d) Testing: The performance of our hybrid approach is evaluated according to the principle of $k$-fold cross-validation where the $k$-folds form a partition of the dataset (with $k=$ 4). Each fold is used exactly once as a validation set during training. In the CAD-120 dataset there are four actors and each fold is associated with one actor. In Watch-n-Patch, the original publication [24] provides one test and training sets, we generate 3 more folds while keeping the actions in the same sequence within the same fold. We obtain the final prediction at the last activation layer, softmax, present in variants 2,3 and 4 described in section $[\mathrm{III}$ and illustrated in Fig. 1

\section{Metrics for Evaluation}

We evaluate the different variants proposed in section III with two metrics. The first one is the accuracy, later called micro-accuracy $(\mu)$, which is defined as follows:

$$
\mu \text {-accuracy }=\frac{\text { number of correct predictions }}{\text { total number of predictions }} .
$$

This measures the ratio of correctly recognized actions to the total number of actions to recognize. In contrast, the second metric called macro-accuracy (M) measures the average of the accuracy for each class. The accuracy of each class is calculated and the macro-accuracy is the average of these accuracies. Macro-accuracy gives the same weight to each class, regardless of the number of samples the class has in the dataset. This makes possible to see if only the most represented classes are correctly recognized or if globally all the classes, including the under-represented ones, are correctly recognized. These two metrics are complementary in performance evaluation for datasets with imbalanced classes.

\section{Results and discussion}

We first compare the individual results of C3D, C3D-GRU and ANBM variants described in section [II] before evaluating their late fusion.

Here we evaluate the contribution of the GRU recurrent layer at the output of C3D to take into account the temporal logic between actions (C3D-GRU). According to Tab. I in Watch-n-Patch we observe, on average, a gain in microaccuracy of +13 percentage points (cf. lines 2 and 3). Regarding CAD-120 dataset, we observe on Tab. II (cf. lines 2 and 3) a gain in micro-accuracy of +4 percentage points thanks to the addition of a GRU layer to the C3D network compared to $\mathrm{C} 3 \mathrm{D}$ alone. Looking in detail at the different confusion matrices obtained on Watch-n-Patch on Fig. 5 and 6 we see that classes that benefit the most are the following: put-back-book, put-down-item and take-item. Indeed the action put-back-book is often preceded by the action read. When the previous action is labeled as read, it reduces and conditions the choice of the following possibilities. The action put-down-item is often preceded by action walk. Indeed, in Watch-n-Patch it 
is a common scenario for a person to walk into the office and put his or her phone on the table. The gains on CAD120 are more modest because for the recurrent layer to bring information, the frozen $\mathrm{C} 3 \mathrm{D}$ network must have learned to recognize classes with a sufficient accuracy.

The C3D-GRU network therefore outperforms C3D and now we are comparing it with our ANBM approach before evaluating their fusion. On the Watch-n-Patch dataset, C3DGRU has a better micro-accuracy than ANBM (+11 percentage points pp) but the improvement of the macro-accuracy is less important $(+5 \mathrm{pp})$, cf. lines 1 and 3 of Tab I As it can be seen on the confusion matrix on Fig. 6, the best detected actions by C3D-GRU are read, walk and leave-office with scores of $1,0.98$, and $0.97 \%$ respectively. These actions represent $49 \%$ of the data (cf. Tab. IIII of the dataset) and contribute more to the micro-accuracy than, for example, turn-off-computer which represents only 3\%. The confusion matrix of Fig. 4 shows us that the ANBM approach outperforms C3D-GRU on 3 classes: play-computer, turn-on-computer and turn-offcomputer. Both approaches perform best on different classes, but the nature of false positives also varies. As we can see from the confusion matrices in Figs. 4 and 6 both approaches have similar performances for the action fetch-book $(0.71 \%$ for ANBM and $0.81 \%$ for C3D-GRU) but the errors differ. Indeed ANBM sometimes detects reach while C3D-GRU detects place instead of fetch-book On CAD-120 the C3DGRU network distinguishes better reaching and placing than ANBM, these two actions represent $45 \%$ of the dataset.

Thus, both datasets C3D-GRU and ANBM bring performances that complement each other. Giving best performances on different classes and different false positive sources of error may be one reason why the fusion using a fully connected layer may capture more information than a simple average of the two outputs. Here we evaluate the benefits that can be derived from their fusion. On CAD-120, the ability of the C3D-GRU network to distinguish reach and place from other classes allows, when merging both approaches, a gain of +4 percentage points in micro-accuracy, see Tab. III. The fusion of C3D-GRU and ANBM improves the recognition of every actions on Watch-n-Patch except the action walk (1) which drops from 0.98 with C3D-GRU to 0.97 as well as actions play-computer (7) and turn-off-computer (9) that also drop by $1 \%$ with C3D-GRU-ANBM-DF fusion as shown by the confusion matrices on Figs. 46 and 7 Overall, predictions fusion increases the micro-accuracy by +6 percentage points with respect to C3D-GRU and by +17 percentage points with respect to ANBM. In the fusion, for actions involving a computer the performances of ANBM are favoured over those of C3D-GRU. The fact that both approaches complement each other is also well exploited when they individually present similar performances for a same class. For example, with the fusion approach the action fetch-book reaches 0.94 whereas with C3D-GRU and ANBM this action was correctly predicted in respectively 0.81 and 0.77 .

Here we propose to evaluate the robustness of this fusion approach on Watch-n-Patch by smoothing or further degrad-

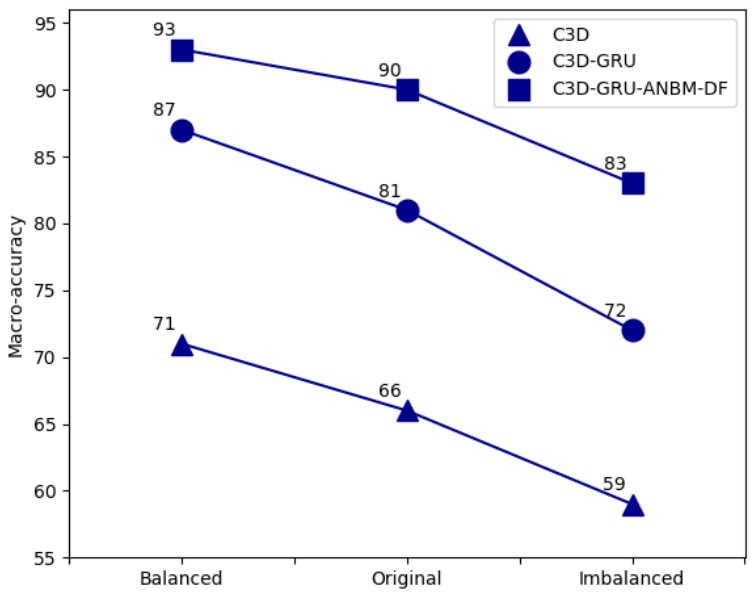

Fig. 3: Macro-accuracy with respect to Watch-n-Patch dataset with classes synthetically augmented in order to decrease or increase the class imbalance.

TABLE IV: Comparison to the literature. Action recognition accuracy on two public datasets: CAD-120 and Watch-n-Patch.

\begin{tabular}{lll} 
Dataset & Approaches & Accuracy \\
\hline CAD-120 & GEPHAPP [28] & 79.4 \\
& ANBM [9] & 82.2 \\
& GPNN [29] & 87.3 \\
\hline & Ours & $\mathbf{8 6 . 1}$ \\
\hline Watch-n-Patch & CaTM [24] & 32.9 \\
& WBTM [30] & 35.2 \\
& PoT [31] & 49.93 \\
& ANBM [9] & 76.4 \\
& GEPHAPP [28] & 84.8 \\
\hline & Ours & $\mathbf{9 3 . 0}$ \\
\hline
\end{tabular}

ing the class imbalance within the dataset. We synthetically augment or degrade the dataset and we re-train the networks C3D, C3D-GRU, C3D-GRU-ANBM-DF to obtain the results presented in Fig. 3. We observe that C3D training is sensitive to the number of samples in the training. We also observe the dependency of C3D-GRU on the result of C3D. Indeed C3DGRU performance drops faster than C3D, because to capture temporal coherence, the previous action must be well detected. When classes are strongly imbalanced, C3D detects poorly some actions and some temporal transitions between action are not modeled. Overall, as expected we note that the fusion of C3D-GRU-ANBM-DF resist more to the degradation of the samples, with a slightly less important slope value.

As shown in Tab. IV] our hybrid fusion strategy allows us to improve our previous performance, while still having near or better than the state of the art performances. We select recent state-of-the-art benchmark approaches and if possible that are evaluated on the same two datasets such as 
Fig. 4: Confusion matrix of ANBM (original test set from Watch-n-Patch). Predictions are on columns are ground truth on rows. [0 - read ; 1 - walk ; 2 - leave-office ; 3 - fetch-book ; 4 - put-back-book ; 5 - put-down-item ; 6 - take-item ; 7 play-computer ; 8 - turn-on-computer ; 9 - turn-off-computer]

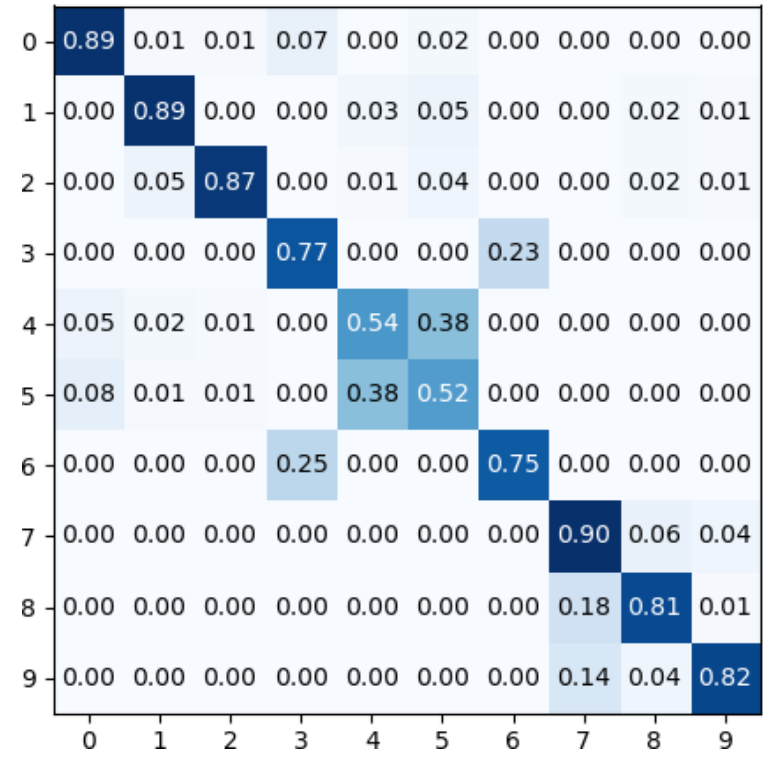

Qi et al. [28]. The two approaches considered in our hybrid fusion strategy take into account the transitions between two successive actions. In CAD-120, the action moving precedes almost all the others, which is not very informative. We may consider to take into account more transitions. It also shows that our merging strategy allows us to surpass the state of the art in action recognition on the Watch-n-Patch dataset by improving action recognition by +8.2 percentage points compared to the approach proposed by Qi et al [28].

A video illustrates our results on video sequences of both datasets is available at the address indicated in the footnote 1

\section{CONCLUSiOn AND Future Work}

In this paper we have compared different approaches for action detection and proposed the addition of a recurrent layer to $\mathrm{C} 3 \mathrm{D}$ to benefit from the temporal relationships between actions. We explored a way to merge at the decision level of data driven and Bayesian-based approaches for action recognition using a dense layer. We experimented with two datasets in the literature presenting an imbalance between their classes, and we show gains in accuracy that are even more significant when the approaches complement each other.

In the perspectives we plan to evaluate our merging approach on the detection of high-level activities composed by the succession of atomic actions. In the future we also plan to further investigate deep learning architectures for automatic action segmentation in order to deal with untrimmed video data.
Fig. 5: Confusion matrix of C3D (original test set from Watchn-Patch). Predictions are on columns are ground truth on rows. [0 - read ; 1 - walk ; 2 - leave-office ; 3 - fetch-book ; 4 put-back-book ; 5 - put-down-item ; 6 - take-item ; 7 - playcomputer ; 8 - turn-on-computer ; 9 - turn-off-computer]

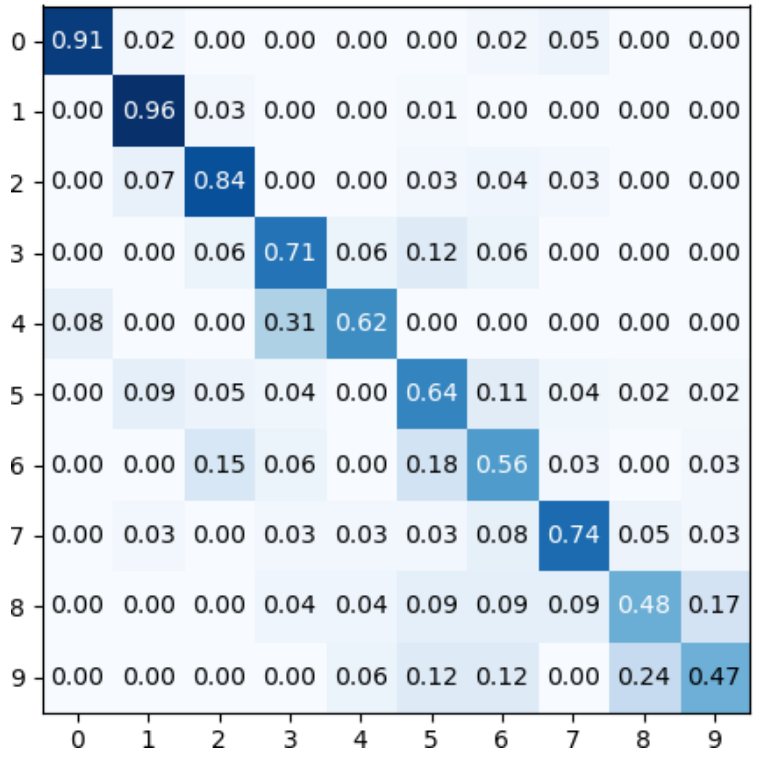

Fig. 6: Confusion matrix of C3D-GRU (original test set from Watch-n-Patch). Predictions are on columns are ground truth on rows. [0 - read ; 1 - walk ; 2 - leave-office ; 3 - fetch-book ; 4 - put-back-book ; 5 - put-down-item ; 6 - take-item ; 7 play-computer ; 8 - turn-on-computer ; 9 - turn-off-computer]

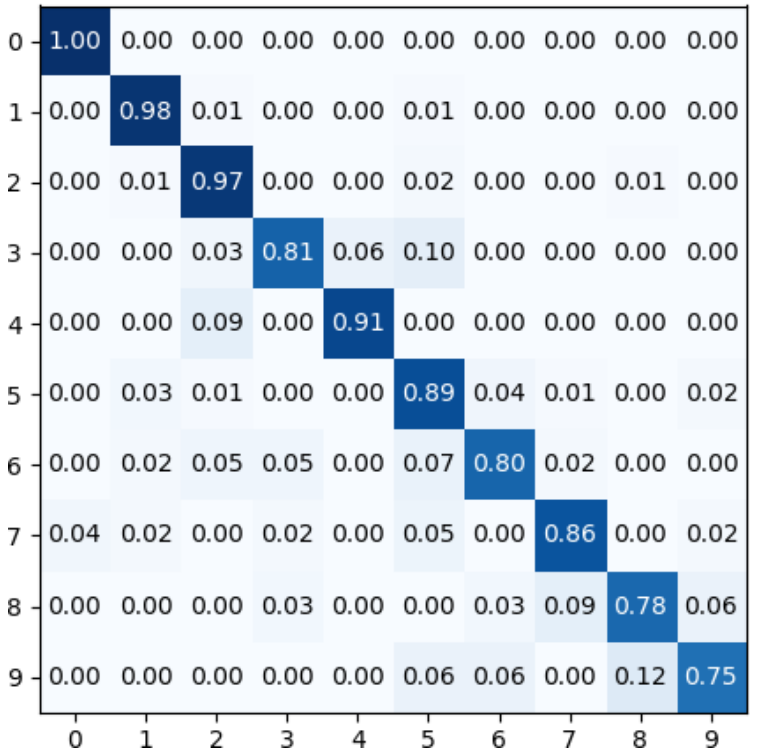


Fig. 7: Confusion matrix of our fusion approach C3D-GRUANBM-DF (original test set from Watch-n-Patch). Predictions are on columns are ground truth on rows. [0 - read ; 1 - walk ; 2 - leave-office ; 3 - fetch-book ; 4 - put-back-book ; 5 put-down-item ; 6 - take-item ; 7 - play-computer ; 8 - turnon-computer ; 9 - turn-off-computer]

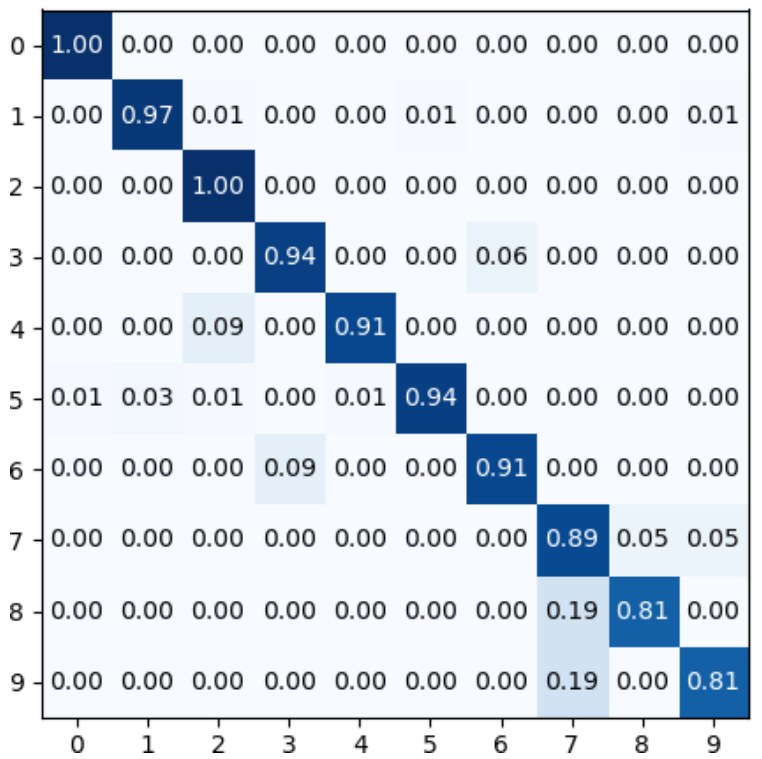

[10] B. Zhou, A. Khosla, A. Lapedriza, A. Oliva, and A. Torralba, "Learning deep features for discriminative localization," in Proceedings of the IEEE conference on computer vision and pattern recognition, pp. 2921-2929, 2016.

[11] M. Oquab, L. Bottou, I. Laptev, and J. Sivic, "Learning and transferring mid-level image representations using convolutional neural networks," in Proceedings of the IEEE conference on computer vision and pattern recognition, pp. 1717-1724, 2014.

[12] M. Everingham, L. Van Gool, C. K. Williams, J. Winn, and A. Zisserman, "The pascal visual object classes (voc) challenge," International journal of computer vision, vol. 88, no. 2, pp. 303-338, 2010.

[13] A. Gupta and L. S. Davis, "Objects in action: An approach for combining action understanding and object perception," in 2007 IEEE Conference on Computer Vision and Pattern Recognition, pp. 1-8, IEEE, 2007.

[14] S. Ji, W. Xu, M. Yang, and K. Yu, "3d convolutional neural networks for human action recognition," IEEE transactions on pattern analysis and machine intelligence, vol. 35, no. 1, pp. 221-231, 2012.

[15] D. Tran, J. Ray, Z. Shou, S.-F. Chang, and M. Paluri, "Convnet architecture search for spatiotemporal feature learning," arXiv preprint arXiv:1708.05038, 2017

[16] G. Varol, I. Laptev, and C. Schmid, "Long-term temporal convolutions for action recognition," IEEE transactions on pattern analysis and machine intelligence, vol. 40, no. 6, pp. 1510-1517, 2017.

[17] K. Liu, W. Liu, C. Gan, M. Tan, and H. Ma, "T-c3d: temporal convolutional $3 \mathrm{~d}$ network for real-time action recognition," in Thirtysecond AAAI conference on artificial intelligence, 2018.

[18] X. Wang, L. Gao, J. Song, and H. Shen, "Beyond frame-level cnn: saliency-aware 3-d cnn with 1 stm for video action recognition," IEEE Signal Processing Letters, vol. 24, no. 4, pp. 510-514, 2016.

[19] N. Lu, Y. Wu, L. Feng, and J. Song, "Deep learning for fall detection: Three-dimensional cnn combined with lstm on video kinematic data," IEEE journal of biomedical and health informatics, vol. 23, no. 1, pp. 314-323, 2018.

[20] G. Yao, X. Liu, and T. Lei, "Action recognition with 3d convnet-gru architecture," in ICRA, pp. 208-213, 2018.

[21] C. Feichtenhofer, A. Pinz, and A. Zisserman, "Convolutional two-stream network fusion for video action recognition," in Proceedings of the IEEE conference on computer vision and pattern recognition, pp. 1933-1941, 2016.

This work has been partially supported by Bpifrance within the French Project LinTO and funded by the French government under the Investments for the Future Program (PIA3).

\section{REFERENCES}

[1] P. Turaga, R. Chellappa, V. S. Subrahmanian, and O. Udrea, "Machine recognition of human activities: A survey," IEEE Transactions on Circuits and Systems for Video technology, vol. 18, no. 11, pp. 14731488, 2008.

[2] T. B. Moeslund, A. Hilton, and V. Krüger, "A survey of advances in vision-based human motion capture and analysis," Computer vision and image understanding, vol. 104, no. 2-3, pp. 90-126, 2006.

[3] D. Tran, L. Bourdev, R. Fergus, L. Torresani, and M. Paluri, "Learning spatiotemporal features with $3 \mathrm{~d}$ convolutional networks," in Proceedings of the IEEE international conference on computer vision, pp. 44894497, 2015.

[4] K. Soomro, A. R. Zamir, and M. Shah, "Ucf101: A dataset of 101 human actions classes from videos in the wild," arXiv preprint arXiv:1212.0402, 2012.

[5] A. Shahroudy, J. Liu, T.-T. Ng, and G. Wang, "Ntu rgb+ d: A large scale dataset for 3d human activity analysis," in Proceedings of the IEEE conference on computer vision and pattern recognition, pp. 1010-1019, 2016.

[6] J. Carreira and A. Zisserman, "Quo vadis, action recognition? a new model and the kinetics dataset," in proceedings of the IEEE Conference on Computer Vision and Pattern Recognition, pp. 6299-6308, 2017.

[7] W. Li, Z. Zhang, and Z. Liu, "Action recognition based on a bag of 3d points," in 2010 IEEE Computer Society Conference on Computer Vision and Pattern Recognition-Workshops, pp. 9-14, IEEE, 2010.

[8] H. S. Koppula, R. Gupta, and A. Saxena, "Learning human activities and object affordances from rgb-d videos," The International Journal of Robotics Research, vol. 32, no. 8, pp. 951-970, 2013.

[9] C. Maurice, F. Madrigal, A. Monin, and F. Lerasle, "A new bayesian modeling for 3d human-object action recognition," in 2019 16th IEEE International Conference on Advanced Video and Signal Based Surveillance (AVSS), pp. 1-8, IEEE, 2019.
22] Y. Fan, X, Lu, D. Li, and Y. Liu, "Video-based emotion recognition using cnn-rnn and c3d hybrid networks," in Proceedings of the 18th ACM International Conference on Multimodal Interaction, pp. 445-450, 2016.

[23] F. Baradel, C. Wolf, and J. Mille, "Human activity recognition with pose-driven attention to rgb," 2018.

[24] C. Wu, J. Zhang, S. Savarese, and A. Saxena, "Watch-n-patch: Unsupervised understanding of actions and relations," in Proceedings of the IEEE Conference on Computer Vision and Pattern Recognition, pp. 4362 4370, 2015.

[25] Z. Cao, T. Simon, S.-E. Wei, and Y. Sheikh, "Realtime multi-person 2 pose estimation using part affinity fields," in Proceedings of the IEEE Conference on Computer Vision and Pattern Recognition, pp. 72917299, 2017.

[26] T.-Y. Lin, M. Maire, S. Belongie, J. Hays, P. Perona, D. Ramanan, P. Dollár, and C. L. Zitnick, "Microsoft coco: Common objects in context," in European conference on computer vision, pp. 740-755, Springer, 2014

[27] W. Liu, D. Anguelov, D. Erhan, C. Szegedy, S. Reed, C.-Y. Fu, and A. C. Berg, "Ssd: Single shot multibox detector," in European conference on computer vision, pp. 21-37, Springer, 2016.

[28] S. Qi, B. Jia, S. Huang, P. Wei, and S.-C. Zhu, "A generalized earley parser for human activity parsing and prediction," IEEE Transactions on Pattern Analysis and Machine Intelligence, 2020.

[29] S. Qi, W. Wang, B. Jia, J. Shen, and S.-C. Zhu, "Learning human-object interactions by graph parsing neural networks," in Proceedings of the European Conference on Computer Vision (ECCV), pp. 401-417, 2018

[30] C. Wu, J. Zhang, B. Selman, S. Savarese, and A. Saxena, "Watchbot: Unsupervised learning for reminding humans of forgotten actions," in 2016 IEEE International Conference on Robotics and Automation (ICRA), pp. 2479-2486, IEEE, 2016.

[31] M. S. Ryoo, B. Rothrock, and L. Matthies, "Pooled motion features for first-person videos," in Proceedings of the IEEE Conference on Computer Vision and Pattern Recognition, pp. 896-904, 2015. 